\title{
DESEMPENHO DOS ÍNDICES DE NESTEROV E FÓRMULA DE MONTE ALEGRE NO DISTRITO DE LICHINGA, NORTE DE MOÇAMBIQUE
}

\author{
PERFORMANCE OF NESTEROV INDEXES AND OF MONTE ALEGRE FORMULA IN LICHINGA \\ DISTRICT, NORTHERN OF MOZAMBIQUE
}

\author{
Aires Afonso Mbanze ${ }^{1}$ Antonio Carlos Batista ${ }^{2}$ Alexandre França Tetto ${ }^{2}$ Axel Misraim Romero ${ }^{3}$ \\ John Mudekwe ${ }^{4}$
}

\begin{abstract}
RESUMO
Este trabalho foi desenvolvido com objetivo de avaliar o desempenho dos índices de Nesterov e Fórmula de Monte Alegre (FMA) no ambiente Lichinga (norte de Moçambique), de modo a sugerir o melhor índice a ser usado nesta região. Para a materialização desta pesquisa, foram usados dados meteorológicos diários (temperatura, precipitação e umidade relativa) e dados de registro de ocorrências dos incêndios florestais de um período de três anos (2010 a 2012). Os dados meteorológicos foram fornecidos pelo Instituto de Investigação Agronômica de Moçambique em Lichinga (IIAM-Lichinga) e o banco de dados de registro de ocorrências de incêndios foi providenciado pelo Centro de Controle e Monitoramento de Incêndios Florestais (CCMIF), da empresa Chikweti Forest of Niassa. Na sequência, foram calculados os índices de perigo de incêndios e o grau de perigo diário para os dois índices, tendo-se observado a predominância do grau de perigo muito alto em quase todos os anos, com um porcentual de 52,65\% para o FMA e 37,96\% para o índice Nesterov. Com valores de Skill Score (SS) de 0,11 e Porcentagem de Sucesso (PS) de 44,82\%, o índice Nesterov mostrou melhor desempenho para o ambiente de Lichinga, ao contrário do FMA que apresentou um fraco desempenho com os valores de 0,07 de SS e 35,62\% de PS.
\end{abstract}

Palavras-chave: incêndios florestais; índices de perigo de incêndios; grau de perigo.

\begin{abstract}
This study was conducted in order to evaluate the performance of Nesterov indexes and of Monte Alegre formula (FMA) in Lichinga environment (northern Mozambique) in order to suggest the best index to be used in this region. To do so, we used daily meteorological data (temperature, precipitation and relative humidity) and registration data of forest fire occurrences in a period of three years $(2010-2012)$. Meteorological data were provided by the Mozambican Agricultural Research Institute of Mozambique in Lichinga (IIAM-Lichinga), whereas the data base of fire occurrence record was provided by the Center for Monitoring and Control of Forest Fires (CCMIF) of the company Chikweti Forest of Niassa After that Then, fire danger indexes and the degree of daily danger were calculated for both indexes, where it was observed the predominance of very high degree of danger in almost every year, with a percentage of $52.65 \%$
\end{abstract}

1 Engenheiro Florestal, MSc., Investigador Assistente do Departamento de Ambiente e Conservação de Recursos Naturais na Universidade Lúrio, Faculdade de Ciências Agrárias, Campus Universitários de Unango/Sanga, Moçambique. Estudante de Doutoramento na Universidade Nova de Lisboa, Faculdade de Economia, Campus de Campolide, P.O. Box 1099-032 Lisboa, Portugual. aires.banze@gmail.com or 26421@novasbe.pt

2 Engenheiro Florestal, Dr., Professor do Departamento de Ciências Florestais, Universidade Federal do Paraná, Av. Lothário Meissner, 900, CEP 80210-170, Curitiba (PR), Brasil. batistaufpr@ufpr.br/ tetto@ufpr.br

3 Engenheiro Florestal, Departamento de Planificação, Centro de Monitoria e Controlo de Incêndios Florestais, Chikweti Forest of Niassa, Chikweti Village, Caixa Postal 264, Bairro Nomba, Lichinga, Moçambique. axel@chikweti.com/ axelrox77@yahoo.com

4 Engenheiro Florestal, Ph.D., Gestor do Departamento de Pesquisa e Desenvolvimento, Chikweti Forest of Niassa, Chikweti Village, Caixa Postal 264, Bairro Nomba, Lichinga, Moçambique. john.mudekwe@chikweti.com 
for the FMA and $37.96 \%$ for Nesterov index. Along with values of Skill Score (SS) of 0.11 Percent Success (PS) of $44.82 \%$, Nesterov index showed best performance for this region unlike FMA, that presented a weak performance with values of 0,07 of SS and $35.62 \%$ of PS.

Keywords: forest fires; fire danger indexes; degree of danger.

\section{INTRODUÇÃO}

A diminuição da cobertura vegetal e a intensificação das atividades humanas resultaram em um aumento no risco de incêndios florestais. Esses eventos, presentes no planeta, desde os tempos mais remotos (RODRIGUEZ; SOARES, 2004), têm causado sérios impactos, sendo que suas ocorrências dependem, entre outros aspectos, da época do ano e da sua localização geográfica (TETTO; BATISTA; PIVOVAR, 2008). Em vários países a ocorrência de incêndios tem se intensificado em função do aumento populacional, acúmulo de material combustível e atividades humanas (SOARES, 2009). Além destes aspectos, a mudança espaço-temporal do clima altera o regime do fogo: padrões, frequência, intensidade e distribuição dos incêndios (KRAWCHUK et al., 2009).

Como forma de predizer o comportamento esperado do fogo para cada dia e as condições dos combustíveis florestais são utilizados índices de perigo de incêndios (HEIKKILÄ; GRÖNQVIST; JURVÉLIUS, 2010). Esses indices são ferramentas que auxiliam no planejamento e supervisão das atividades de controle e combate aos incêndios florestais (SOARES, 1998).

O conhecimento do índice de perigo é fundamental dentro de um plano de prevenção e combate aos incêndios florestais, por possibilitar a adoção de medidas preventivas em bases mais técnicas e econômicas. Isto porque no controle de incêndios, a concentração de muitos recursos torna-se excessivamente cara e a racionalização excessiva dos mesmos pode acarretar altos prejuízos (SOARES; BATISTA, 2007; SOARES; BATISTA; NUNES, 2008; RODRÍGUEZ, 2010).

Segundo Soares e Batista (2007), os índices de perigo de incêndios mais difundidos, principalmente no Brasil, são os índices de Angstron (B), Nesterov (G), Fórmula de Monte Alegre (FMA) e Fórmula de Monte Alegre Alterada (FMA ${ }^{+}$). Tetto (2012) afirma que o FMA foi o primeiro índice de perigo desenvolvido no Brasil, por Ronaldo Viana Soares em 1972. Este índice tem apresentado um bom desempenho não só em regiões de clima semelhante, mas também em várias outras regiões, justificando-se em alguns casos apenas o ajuste das classes de perigo.

$\mathrm{Na}$ África, o índice mais difundido é o índice de Nesterov, embora em Moçambique também se utilize com frequência a FMA. No norte de Moçambique é comum o uso da previsão meteorológica como forma de anúncio do grau de perigo dos incêndios e planificação das operações de prevenção e combate aos incêndios florestais. Embora exista uma correlação entre as variáveis meteorológicas e o grau de perigo de incêndios, Soares e Batista (2007); Heikkilä, Grönqvist, Jurvélius (2010); Soares, Batista, Nunes (2008) e Tetto (2012) deixaram claro que essa relação não é assim tão clara, ao ponto de garantir eficiência na previsão de ocorrência dos incêndios. O desenvolvimento dos índices de perigo de incêndio e a adequação para uma determinada região onde estes vão ser introduzidos, é a forma correta de fazer a predição da ocorrência dos incêndios florestais. $\mathrm{O}$ fraco aproveitamento desta ferramenta, preciosa para planejamento das operações de prevenção e combate aos incêndios florestais, pelas empresas do setor florestal e outras instituições relacionadas, na província do Niassa, provavelmente esteja primeiramente relacionado com o desconhecimento da sua relevância, seguido pela negligência e por falta de pessoal com capacidade para desenvolver e implementar os índices de perigo de incêndios.

Este trabalho objetivou avaliar o desempenho dos índices de perigo de Nesterov e FMA nas condições do distrito de Lichinga, como forma de subsidiar as empresas florestais que operam nesta região, particularmente a Empresa Chikweti Forest of Niassa, na previsão da ocorrência dos incêndios florestais.

\section{MATERIAL E MÉTODOS}

\section{Caracterização da área de estudo}

O estudo foi conduzido do distrito de Lichinga, província do Niassa, que se localiza no norte de 
Moçambique, nas coordenadas $13^{\circ} 23^{\prime} 48^{\prime \prime}$ S e $35^{\circ} 13^{\prime} 43^{\prime \prime}$ E. A altitude da região varia de 1000 a $1300 \mathrm{~m}$ (Figura 1). A área faz parte de um extenso corredor de cultivo de Pinus sp e Eucalyptus sp, que começaram a ser estabelecidos no ano 2005.

De acordo com a classificação de Köppen, o clima da região é temperado úmido (Cwb), com duas estações bem distintas (verões temperados e chuvosos e invernos secos e frios). A região apresenta precipitação média anual que varia de 1000 a $1500 \mathrm{~mm}$ e a temperatura média anual oscila de 20 a $23^{\circ} \mathrm{C}$ (MOÇAMBIQUE, 2005).

\section{Coleta de dados}

Os dados de ocorrências de incêndios florestais do distrito de Lichinga, dos anos de 2010 a 2013, foram fornecidos pelo Centro de Controle e Monitoramento de Incêndios Florestais (CCMIF) da empresa Chikweti Forest of Niassa. No ano 2010, a empresa Chikweti Forest of Niassa criou um Centro de Controle e Monitoramento de Incêndios Florestais (CCMIF), no qual se faz o acompanhamento das ocorrências dos incêndios, via rádio, da ignição, detecção, deslocamento das equipes, combate, até o momento de avaliação dos danos após o fogo, durante 24 horas dia, e em todos os dias da semana. Todos os dados de ocorrência são registrados, inclusive dados adicionais de avaliação após a ocorrência do incêndio.

As variáveis meteorológicas diárias de temperatura (máxima e mínima), precipitação e umidade relativa, referentes ao período de janeiro de 2009 a dezembro de 2012, foram registradas às 13 horas, na estação meteorológica do Instituto de Investigação Agronômica de Moçambique (IIAM) do distrito de Lichinga, sendo posteriormente disponibilizadas para a realização desta pesquisa.

\section{Análise de dados}

Com o intuito de determinar o grau de perigo dos índices Nesterov e FMA, foram calculados os índices de perigo com o programa Excel 2007, utilizando as fórmulas (1) a (3), que se seguem:

$$
G=\sum_{i=1}^{n} d i . T i
$$

Em que: $G=$ índice de Nesterov; $d=$ deficit de saturação do ar em milibar; $T=$ temperatura do ar em ${ }^{\circ} \mathrm{C}$; $n=$ número de dias sem chuva maior que $10,0 \mathrm{~mm}$;
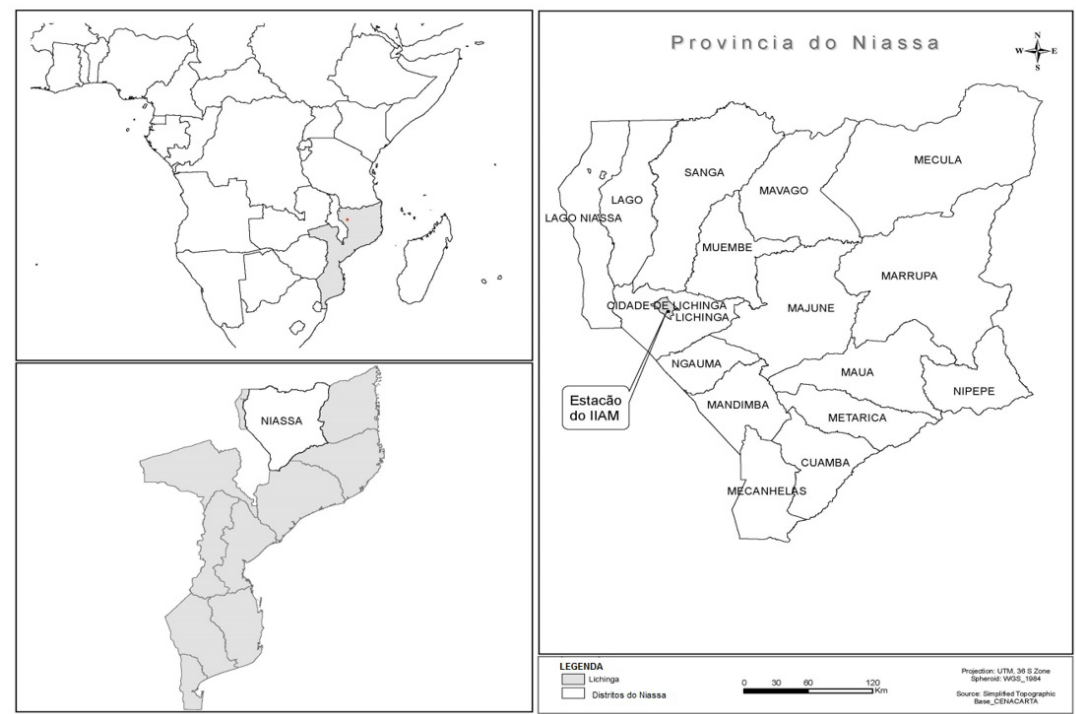

FIGURA 1: Localização da área de estudo.

FIGURE 1: Study site location. 
O deficit de saturação do ar foi calculado com auxílio da fórmula:

$$
d=E(1-H / 100)
$$

Em que: $d=$ deficit de saturação do ar em milibar; $E$ = pressão máxima de vapor de água em milibar; $H=$ umidade relativa do ar em $\%$.

A equação básica da Fórmula de Monte Alegre (FMA) é dada por:

$$
F M A=\sum_{i=1}^{n}(100 / H)
$$

Em que: $F M A=$ Fórmula de Monte Alegre; $H=$ umidade relativa do ar em \%, medida às 13 horas; $n=$ número de dias sem chuva maior que $13 \mathrm{~mm}$.

Ambos os índices (G e FMA) são acumulativos, por isso estão sujeitos às restrições da precipitação (NUNES, 2005; SOARES; BATISTA, 2007; BORGES et al., 2011; TETTO, 2012; RODRÍGUES, 2012). A interpretação do grau de perigo, estimado pelo índice de Nesterov ou FMA, é feito por meio de uma escala de perigo conforme representado na Tabela 1.

Após a determinação dos índices de perigo e as classes de grau de perigo, foram quantificados e analisados os números de dias para cada classe de grau de perigo e os percentuais que os mesmos representam em cada classe de grau de perigo. O desempenho dos índices foi analisado usando a metodologia conhecida por Skill Score (ou desempenho do índice), aplicada por Sampaio (1999). Essa metodologia baseia-se em uma tabela de contingência, que contém os valores observados e os valores previstos para um evento em uma população ou determinado período de tempo, como está ilustrado nas Tabelas 2 e 3 .

Posteriormente, com auxílio dos resultados das Tabelas 2 e 3, foram calculadas as variáveis necessárias para o cálculo do Skill Score e porcentagem de sucesso: $\mathrm{N}=$ número total de observações $(N=a+b+c+d) ; \mathrm{G}=$ número de acertos na previsão $(G=a+d) ; \mathrm{H}=$ número esperado de acertos $(H=N(1-p) \cdot(1-q)+N \cdot p \cdot q)$.

Em que: $p=N 1 / N ; q=N 2 / N$;SS $($ Skill Score $)=(G-H)(N-H) ; \mathrm{PS}=($ Porcentagem de Sucesso) $=100 . G / N$

TABELA 1: Escala de perigo dos índices de Nesterov e FMA.

TABLE 1: Danger scale of Nesterov indexes and FMA.

\begin{tabular}{lcccc}
\hline & VALORES & \multicolumn{3}{c}{ GRAU DE PERIGO } \\
\hline \multicolumn{1}{c}{ G } & FMA & G & FMA & DESIGNAÇÃO \\
\hline$\leq 300$ & $\leq 3,0$ & Nenhum risco & Nulo & 1 \\
301 a 500 & 3,1 a 8,0 & Risco pequeno & Pequeno & 2 \\
501 a 1000 & 8,1 a 20,0 & Risco médio & Médio & 3 \\
1001 a 4000 & 20,1 a 24,0 & Grande risco & Alto & 4 \\
$>4000$ & $>24,0$ & Perigosíssimo & Muito alto & 5 \\
\hline
\end{tabular}

Em que: FMA = Fórmula de Monte Alegre; G = índice de Nesterov. 
TABELA 2: Tabela de contingência.

TABLE 2: Contingence table.

\begin{tabular}{llccc}
\hline \multirow{2}{*}{ Evento } & \multicolumn{2}{c}{ Observado } & \multirow{2}{*}{ Total previsto } \\
\cline { 3 - 4 } & & Incêndio & Não incêndio & \\
\hline Previsto & Incêndio & $\mathrm{a}$ & $\mathrm{b}$ & $\mathrm{N} 2=\mathrm{a}+\mathrm{b}$ \\
& Não incêndio & $\mathrm{c}$ & $\mathrm{d}$ & $\mathrm{N} 4=\mathrm{c}+\mathrm{d}$ \\
\multicolumn{2}{l}{ Total observado } & $\mathrm{N} 1=\mathrm{a}+\mathrm{c}$ & $\mathrm{N} 3=\mathrm{b}+\mathrm{d}$ & $\mathrm{N}=(\mathrm{a}+\mathrm{b})+(\mathrm{c}+\mathrm{d})$ \\
\hline
\end{tabular}

TABELA 3: Cálculo da tabela de contingência.

TABLE 3: The calculation of the contingence table.

\begin{tabular}{llccc}
\hline \multirow{2}{*}{ Evento } & \multicolumn{2}{c}{ Observado } & \multirow{2}{*}{ Total previsto } \\
\cline { 3 - 4 } & & Incêndio & Não incêndio & \\
\hline Previsto & Incêndio & $\mathrm{a} /(\mathrm{a}+\mathrm{c})$ & $\mathrm{b} /(\mathrm{b}+\mathrm{d})$ & 1 \\
& Não incêndio & $\mathrm{c} /(\mathrm{a}+\mathrm{c})$ & $\mathrm{d} /(\mathrm{b}+\mathrm{d})$ & 1 \\
\multicolumn{2}{l}{ Total observado } & 1 & 1 & $\mathrm{~N}=(\mathrm{a}+\mathrm{b})+(\mathrm{c}+\mathrm{d})$ \\
\hline
\end{tabular}

O Skill Score (SS) é a razão da diferença entre os acertos na previsão (G) e o número esperado de acertos $(\mathrm{H})$ com a diferença entre os números de dias observados $(\mathrm{N})$ e o número de dias com previsão de acertos (H). Na sequência, foram definidos os pontos que indicam a ocorrência e não ocorrência dos incêndios, com base nas escalas das fórmulas de Nesterov e FMA. São considerados como não indicativo da probabilidade de ocorrência dos incêndios, os graus de perigo "nulo" e "pequeno" e, como indicativos da probabilidade de ocorrência dos incêndios os graus de perigo "médio", "alto" e "muito alto". A partir desta definição, foram calculados o Skill Score e as porcentagens de sucesso (NUNES, 2008).

\section{RESULTADOS E DISCUSSÃO}

\section{Comportamento do grau de perigo de incêndios ao longo dos anos}

A influência dos valores da precipitação média mensal no comportamento do grau de perigo mensal permitiu agrupar os meses do ano em função do grau de perigo de ocorrência dos incêndios (Tabela 4).

Quando os valores da precipitação média diária foram inferiores a um milímetro, o grau de perigo da FMA foi muito alto e isso aconteceu nos meses de maio a outubro. Quando a precipitação média diária foi igual ou superior a um milímetro, o grau de perigo variou de pequeno a alto, isso ocorreu nos meses de dezembro a abril.

Quanto ao índice de Nesterov $(\mathrm{G})$, os meses de dezembro a abril apresentaram valores que variaram de nulo a pequeno. Nesses meses, os valores da precipitação média diária foram iguais ou inferiores a dois milímetros. De maio a novembro, os valores dos índices de perigo variaram de médio a muito alto e a precipitação média mensal foi inferior a 2 milímetros. 
TABELA 4: Agrupamento dos meses do ano em função do grau de perigo e da média mensal da precipitação. TABLE 4: Grouping, months of the year depending on the degree of hazard and the average of monthly rainfall.

\begin{tabular}{lccc}
\hline & PM $(\mathrm{mm})$ & GP & MESES \\
\hline \multirow{2}{*}{ FMA } & $<1$ & Muito alto & Maio - Outubro \\
& $\geq 1$ & Pequeno - Alto & Novembro - Abril \\
\hline \multirow{2}{*}{ G } & $\geq 2$ & Nulo - Pequeno & Dezembro - Abril \\
& $<2$ & Médio - Muito alto & Maio - Novembro \\
\hline
\end{tabular}

Em que: $\mathrm{FMA}=$ Fórmula de Monte Alegre; $\mathrm{G}$ = índice de Nesterov; $\mathrm{PM}=$ precipitação média diária em milímetros e GP = grau de perigo.

\section{Números de dias previstos para cada classe de grau de perigo}

$\mathrm{Na}$ Tabela 5 encontram-se representados os valores e porcentuais dos números de dias previstos em cada classe de perigo de incêndios. Observa-se que o maior número de dias para ocorrência dos incêndios foi na classe de grau de perigo muito alto, distribuindo-se em 577 e 416 dias para os índices de FMA e Nesterov, respectivamente. $\mathrm{O}$ ano de 2012 foi o que apresentou o maior número de dias com grau de perigo muito alto (classe 5) para o FMA, com 209 dias e um porcentual de 57,10\%. O índice de Nesterov apresentou um grande número de dias com grau de perigo muito alto não apenas no ano 2012, mas também no ano 2011, ambos os anos com 151 ocorrências, o que correspondeu a 41,37 e 41,26\% para os anos 2011 e 2012, respectivamente. Em seguida, a classe de perigo que se destacou foi a de grau pequeno para o FMA, com um total de 196 ocorrências (17,88\% do total), enquanto que para o índice de Nesterov foi o grau de perigo nulo com um total de 374 , ou $34,12 \%$ do total.

A predominância do número de dias com grau de perigo muito alto também foi observado por

TABELA 5: Porcentagem e número de dias previstos em cada classe de grau de perigo(GP).

TABLE 5: Percentage and number of days expected for each degree class of fire danger(GP).

\begin{tabular}{|c|c|c|c|c|c|c|c|c|}
\hline \multirow{3}{*}{ GP } & \multicolumn{4}{|c|}{2010} & \multicolumn{4}{|c|}{2011} \\
\hline & \multicolumn{2}{|c|}{ FMA } & \multicolumn{2}{|c|}{ G } & \multicolumn{2}{|c|}{ FMA } & \multicolumn{2}{|c|}{$G$} \\
\hline & $\mathrm{N}$ & $\%$ & $\mathrm{~N}$ & $\%$ & $\mathrm{~N}$ & $\%$ & $\mathrm{~N}$ & $\%$ \\
\hline 1 & 29 & 7,95 & 126 & 34,52 & 42 & 11,51 & 130 & 35,62 \\
\hline 2 & 64 & 17,53 & 12 & 3,29 & 69 & 18,90 & 22 & 6,03 \\
\hline 3 & 52 & 14,25 & 16 & 4,38 & 41 & 11,23 & 26 & 7,12 \\
\hline 4 & 39 & 10,68 & 97 & 26,58 & 26 & 7,12 & 36 & 9,86 \\
\hline 5 & 181 & 49,59 & 114 & 31,23 & 187 & 51,23 & 151 & 41,37 \\
\hline TOTAL & 365 & 100,00 & 365 & 100,00 & 365 & 100,00 & 365 & 100,00 \\
\hline GP & \multicolumn{4}{|c|}{2012} & \multicolumn{4}{|c|}{$2010-2012$} \\
\hline 1 & 41 & 11,20 & 118 & 32,24 & 112 & 10,22 & 374 & 34,12 \\
\hline 2 & 63 & 17,21 & 15 & 4,10 & 196 & 17,88 & 49 & 4,47 \\
\hline 3 & 35 & 9,56 & 13 & 3,55 & 128 & 11,68 & 55 & 5,02 \\
\hline 4 & 18 & 4,92 & 69 & 18,85 & 83 & 7,57 & 202 & 18,43 \\
\hline 5 & 209 & 57,10 & 151 & 41,26 & 577 & 52,65 & 416 & 37,96 \\
\hline TOTAL & 366 & 100,00 & 366 & 100,00 & 1096 & 100,00 & 1096 & 100,00 \\
\hline
\end{tabular}

Em que: FMA = Fórmula de Monte Alegre; $\mathrm{G}$ = índice de Nesterov; $\mathrm{N}=$ número de dias previstos; \% = porcentagem de dias previstos. 
Sampaio (1999), Nunes (2005), Nunes et al. (2010), Borges et al. (2011), Rodrígues (2012) e Tetto (2012), não obstante o segundo maior número de dias ter coincidido com o grau de perigo alto para quase todos os autores citados. No que se refere ao índice de Nesterov, Rodrígues (2012), analisando dados de 20.191 dias em Pinar del Río (Cuba), observou a predominância da classe de perigo muito alto em 1.756 dias, o equivalente a um porcentual de $80,15 \%$, seguida pela classe de grau alto em 169 dias, o equivalente a $7,71 \%$. Entretanto, os valores encontrados por esses autores não divergem muito dos encontrados neste trabalho, pelo menos no que diz respeito à predominância do grau de perigo muito alto para os dois índices.

Segundo Nunes et al. (2010), a distribuição do número de dias previstos, encontrado neste estudo para a FMA em todos os anos, é uma condição não desejada para a ocorrência de incêndios, visto que o comportamento do número de dias previstos não segue uma tendência crescente, da classe nulo para muito alto, que é o comportamento esperado para essa variável, indicando que a FMA precisa ser ajustada.

\section{Desempenho dos índices Nesterov e FMA}

Para avaliar o desempenho dos índices (Skill Score e porcentagem de sucesso), foi criada uma tabela de contingência (Tabela 6), com valores do número de dias que os índices previram a ocorrência ou não ocorrência de incêndios (valores previstos) e o número de dias nos quais verdadeiramente o evento ocorreu (valores observados).

Observa-se que no primeiro ano, os dois índices falharam ao prever a não ocorrência de incêndios em 138 e 93 dias para os índices de Nesterov e FMA, respetivamente, mas tendo observado a ocorrência de dois incêndios no mês de novembro. Como já era previsto pelo número de dias em que predominou o grau de perigo alto e muito alto nos dois índices, o índice G previu menor número de dias (227 dias) de ocorrência de incêndios, contrariamente ao FMA, que no mesmo ano previu maior número de dias de

TABELA 6: Valores de contingência (observados e previstos) para os índices Nesterov (G) e FMA. TABLE 6: Contingency values (observed and predicted) for the indexes of Nesterov (G) and FMA

\begin{tabular}{|c|c|c|c|c|}
\hline \multirow{2}{*}{ Anos } & \multirow{2}{*}{ Índices } & \multirow{2}{*}{ Condições } & \multicolumn{2}{|c|}{$\mathrm{N}^{\circ}$ de dias } \\
\hline & & & Observados & Previstos \\
\hline \multirow{4}{*}{2010} & \multirow{2}{*}{$\mathrm{G}$} & Incêndio & 52 & 227 \\
\hline & & Não incêndio & 2 & 138 \\
\hline & \multirow{2}{*}{ FMA } & Incêndio & 52 & 272 \\
\hline & & Não Incêndio & 2 & 93 \\
\hline \multirow{4}{*}{2011} & \multirow{2}{*}{$\mathrm{G}$} & Incêndio & 36 & 212 \\
\hline & & Não incêndio & 3 & 153 \\
\hline & \multirow{2}{*}{ FMA } & Incêndio & 39 & 254 \\
\hline & & Não incêndio & 0 & 111 \\
\hline \multirow{4}{*}{2012} & \multirow{2}{*}{$\mathrm{G}$} & Incêndio & 38 & 233 \\
\hline & & Não incêndio & 0 & 132 \\
\hline & \multirow{2}{*}{ FMA } & Incêndio & 38 & 262 \\
\hline & & Não incêndio & 0 & 104 \\
\hline \multirow{4}{*}{$2010-2012$} & \multirow{2}{*}{$\mathrm{G}$} & Incêndio & 126 & 672 \\
\hline & & Não incêndio & 5 & 424 \\
\hline & \multirow{2}{*}{ FMA } & Incêndio & 129 & 788 \\
\hline & & Não incêndio & 2 & 308 \\
\hline
\end{tabular}

Em que: $\mathrm{FMA}=$ Fórmula de Monte Alegre; $\mathrm{G}$ = índice de Nesterov. 
ocorrência (272 dias), mas apenas observou a ocorrência de 52 incêndios. Assim sendo, não se pode afirmar que os índices falharam na predição de ocorrência dos incêndios, visto que eles previram a ocorrência dos incêndios utilizando as variáveis meteorológicas. Evidentemente que o ideal seria utilizar um índice que indicasse o grau de perigo, levando em consideração a probabilidade de um ser humano atear fogo em um determinado local da área de estudo, uma vez que na área de estudo a maior parte dos incêndios é devido a causas antrópicas. No ano seguinte, o índice $G$ falhou ao prever a não ocorrência de incêndios em 153 dias, tendo sido registrados três incêndios; enquanto a FMA mostrou melhor desempenho, uma vez que em nenhum dia que foi previsto a não ocorrência de incêndio, eles foram observados. Para o ano 2012, ambos os índices mostraram um bom desempenho ao preverem a não ocorrência de incêndios em 132 e 104 dias, períodos em que não foram observadas ocorrências.

No geral, os dois índices previram maior número de dias com ocorrência de incêndios, 672 e 788 dias para os índices G e FMA, respectivamente. Essa tendência também foi observada por Tetto (2012), para o índice FMA; Rodrígues (2012), para os índices FMA, FMA ${ }^{+}$e Nesterov; Borges et al. (2011), para os índices Nesterov, FMA e FMA FMA. Mas uma tendência contrária, também foi encontrada por Nunes (2005), para os modelos ajustados FMAVE1 e FMAVE2.

Na Figura 2 estão representados os valores de Skill Score (SS) e Porcentagem de Sucesso (PS) calculados a partir dos valores apresentados na Tabela 6. Observa-se que os valores obtidos para o índice $\mathrm{G}$ apresentaram melhores resultados em todos os anos estudados, tanto para o SS como para a PS, comparativamente ao FMA. No ano 2010, os valores de $S S$ encontrados foram 0,12 e 0,07 e os de PS foram 45,35 e 34,61\% para o índice G e FMA respectivamente. Nos anos seguintes, os valores foram: 0,10 e 0,08 para $S S$ e 46,78 e $37,13 \%$ para o PS; 0,09 e 0,07 para $S S$ e 42,18 e $35,15 \%$ para a PS e 0,11 e 0,07 para o $S S$ e 44,82 e 35,62\% de PS, para os anos 2011, 2012 e 2010 - 2012, respectivamente. O índice G apresentou melhor desempenho no ano 2011 se comparado com os demais anos.

Borges et al. (2011), avaliando o desempenho de três índices (FMA, $\mathrm{FMA}^{+}$e Nesterov) no norte do estado de Espírito Santo, encontraram que o FMA demostrou pior desempenho com valores de 0,0945

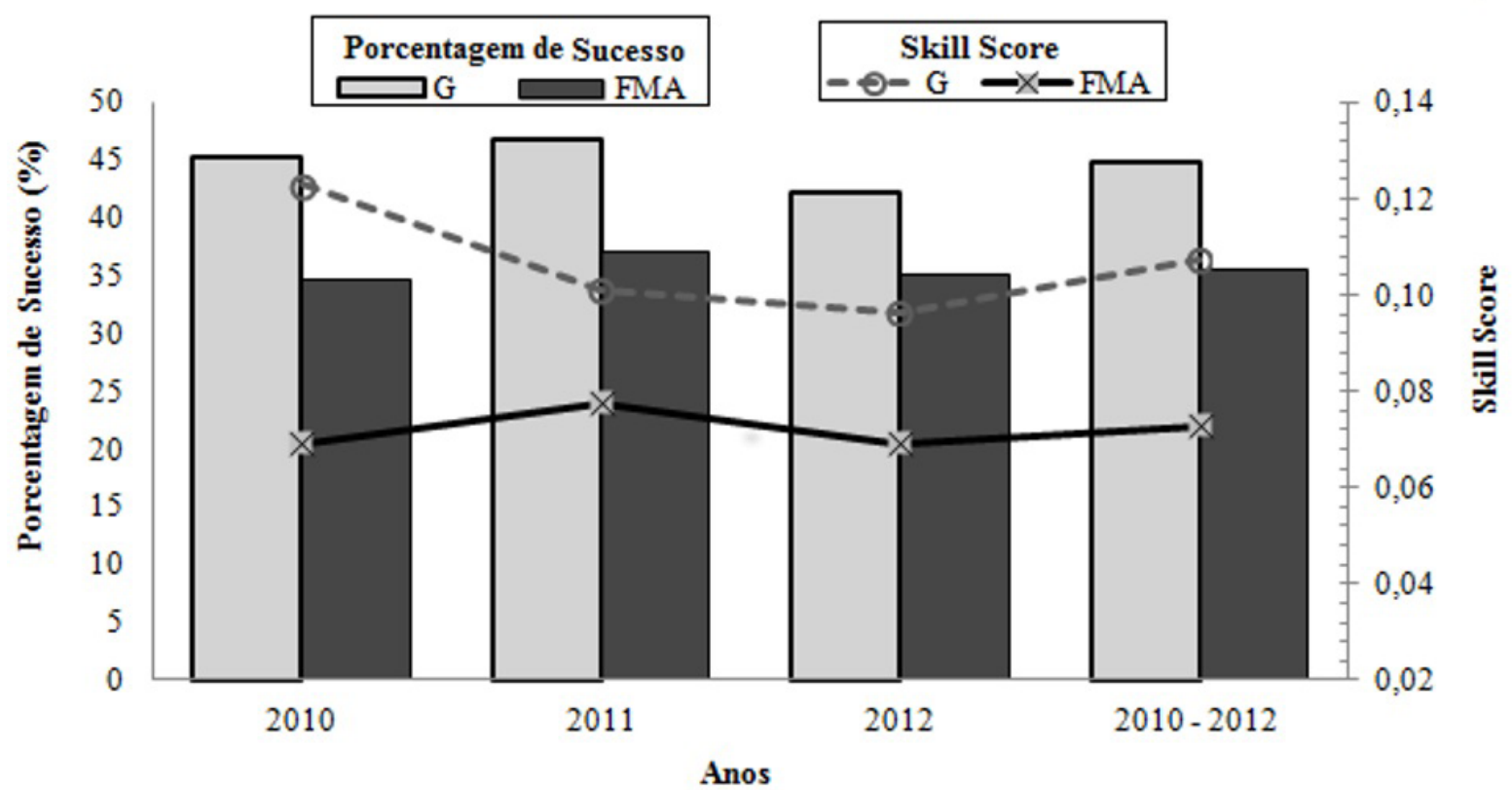

FIGURA 2: Valores de Skill Score e de porcentagem de sucesso para os índices Nesterov (G) e FMA. FIGURE 2: Values of Skill Score and percent of success for Nesterov (G) and FMA indexes. 
e $38,54 \%$ para $S S$ e PS, respectivamente, enquanto que o índice de Nesterov mostrou-se como segundo melhor índice com valores de 0,12 e $46,75 \%$ para o SS e PS, respectivamente. Pesquisa realizada por Sampaio (1999), no período de 1984 a 1995, para a região de Agudos - SP, encontrou o valor médio da porcentagem de sucesso da FMA e do Nesterov iguais a 36,92 e 50,47\%, respectivamente; enquanto o Skill Score destes índices foram 0,0607 e 0,1024. Vários autores, tais como Rodríguez (2012), Tetto (2012), Borges et al. (2011) e Nunes et al. (2010) encontraram valores do SS inferiores a 0,01 e de PS iguais ou inferiores a $40 \%$ para o índice FMA. Esses valores são semelhantes aos encontrados neste trabalho em todos os anos estudados, visto que os valores de $S S$ para o FMA variaram de 0,0695 a 0,0778 e os de PS variaram de 34,61 a $35,62 \%$. Um fato bastante curioso é que na maior parte dos estudos realizados, incluindo o índice $\mathrm{FMA}^{+}$, este quase sempre mostrou desempenho superior se comparado aos demais índices (RODRÍGUEZ, 2012; BORGES et al., 2011; NUNES et al., 2010), sugerindo a necessidade que em trabalhos futuros sejam feitas comparação de desempenho dos índices para o ambiente de Lichinga, com a Fórmula de Monte Alegre Alterada $\left(\mathrm{FMA}^{+}\right)$.

\section{CONCLUSÃO}

A predominância do grau de perigo muito alto em um período bastante longo (maio a outubro) para o índice de perigo FMA e a predominância do grau de perigo que variou de médio a muito alto de maio a novembro para o índice Nesterov, são indicativos de que a área estudada é bastante propensa à ocorrência de incêndios florestais.

$\mathrm{O}$ índice de Nesterov apresentou melhor desempenho para o ambiente de Lichinga, sugerindo a implementação deste para previsão dos incêndios florestais, enquanto não forem desenvolvidos ou ajustados outros índices.

\section{REFERÊNCIAS}

BORGES, T. S. et al. Desempenho de alguns índices de risco de incêndios em plantios de Eucalyptus no norte do Espírito Santo. Floresta e Ambiente, Rio de Janeiro, v. 18, n. 2, p. 153-159, 2011.

HEIKKILÄ, T. V.; GRÖNQVIST, R.; JURVÉLIUS, M. Wildland fire management: handbook for trainers. Helsinki: Ministry for Foreign Affairs of Finland, Development Policy Information Unit, 2010. 248 p.

KRAWCHUK, M. A. et al. Global pyrogeography: the current and future distribution of wildfire. PloS One, São Francisco, v. 4, n. 4, 2009.

MOÇAMBIQUE. Ministério da Administração Estatal. Perfil do distrito de Lichinga. Província de Niassa: MAE, 2005. $54 \mathrm{p}$.

NUNES, J. R. S. FMA ${ }^{+}$- um novo índice de perigo de incêndios florestais para o estado do Paraná - Brasil. 2005. 169 f. Tese (Doutorado em Ciências Florestais) - Setor de Ciências Agrárias, Universidade Federal do Paraná, Curitiba, 2005.

NUNES, J. R. S. Efeitos das mudanças climáticas sobre os índices de perigo de incêndios florestais. Curitiba: UFPR, 2008. 57 p.

NUNES, J. R. S. et al. Desempenho da Fórmula de Monte Alegre (FMA) e da Fórmula de Monte Alegre Alterada $\left(\mathrm{FMA}^{+}\right)$no Distrito Florestal de Monte Alegre. Revista Floresta, Curitiba, v. 40, n. 2, p. 319-326, 2010.

RODRÍGUEZ, M. P. R. Desempeño de los índices de Nesterov, Fórmula de Monte Alegre y Fórmula de Monte Alegre Alterada en la empresa Forestal Macurije Pinar del Río, Cuba. 2012. 109 f. Tese (Pós-doutorado em Engenharia Florestal) - Setor de Ciências Agrárias, Universidade Federal do Paraná, Curitiba, 2012.

RODRÍGUEZ, M. P. R. Manejo del fuego. Havana: Editorial Félix Varela, 2010. 230 p.

RODRIGUEZ, M. P. R.; SOARES, R. V. Análisis comparativo entre los incêndios forestales en Monte Alegre, Brasil y Pinar del Río, Cuba. Floresta, Curitiba, v. 34, n. 2, p. 101-107, 2004.

SAMPAIO, O. B. Análise da eficiência de quatro índices, na previsão de incêndios florestais para a região de Agudos - SP. 1999. 157 f. Tese (Doutorado em Engenharia Florestal) - Universidade Federal do Paraná, Curitiba, 1999. 
SOARES, R. V. Desempenho da "Fórmula de Monte Alegre" índice brasileiro de perigo de incêndios florestais. Revista Cerne, Lavras, v. 4, n. 1, p. 87-99, 1998.

SOARES, R. V. Estatísticas dos incêndios florestais no Brasil. In: SOARES, R. V.; BATISTA, A. C.; NUNES, J. R. S. Incêndios florestais no Brasil: o estado da arte. Curitiba: UFPR, 2009. p. 1-20.

SOARES, R. V.; BATISTA, A. C. Incêndios florestais: controle, efeito e usos do fogo. Curitiba: UFPR, 2007. $250 \mathrm{p}$.

SOARES, R. V.; BATISTA, A. C.; NUNES, J. R. S. Manual de prevenção e combate a incêndios florestais. 2. ed. Curitiba: UFPR, 2008. 55 p.

TETTO, A. F. Comportamento histórico dos incêndios florestais na Fazenda Monte Alegre no período de 1965 a 2009. 2012. 115 f. Tese (Doutorado em Engenharia Florestal) - Setor de Ciências Agrárias, Universidade Federal do Paraná, Curitiba, 2012.

TETTO, A. F.; BATISTA, A. C.; PIVOVAR, C. Manejo da biomassa pós-colheita como forma de prevenção aos incêndios florestais. In: SEMINÁRIO DE ATUALIZAÇÃO EM SISTEMAS DE COLHEITA E TRANSPORTE FLORESTAL, 15., 2008, Curitiba. Anais... Curitiba: FUPEF, 2008. p. 286. 